\title{
Nanotheranostics
}

2019; 3(4): 299-310. doi: 10.7150/ntno.35342

Research Paper

\section{Theranostic nanoparticles enhance the response of glioblastomas to radiation} \author{
M. Loadman ${ }^{3}$, Robert A. Falconer ${ }^{3}$, Sudip Mukherjee ${ }^{1}$, Frederick T. Chin ${ }^{1 凶}$, Heike E. Daldrup-Link ${ }^{1 凶}$ \\ 1. Department of Radiology, Molecular Imaging Program at Stanford, Stanford University, Stanford, CA, USA \\ 2. Department of Radiation Oncology, Stanford University, Stanford, CA, USA \\ 3. Institute of Cancer Therapeutics, Faculty of Life Sciences, University of Bradford, Bradford, UK \\ 4. Department of Neuropathology, School of Medicine, Technical University of Munich, Munich, Germany \\ ${ }^{*}$ Co-first author
}

Wei $\mathrm{Wu}^{1 *}$, Jessica L. Klockow ${ }^{1 *}$, Suchismita Mohanty ${ }^{1}$, Kimberly S. Ku ${ }^{1}$, Maryam Aghighi ${ }^{1}$, Stavros Melemenidis ${ }^{2}$, Zixin Chen ${ }^{1}$, Kai Li ${ }^{1}$, Goreti Ribeiro Morais ${ }^{3}$, Ning Zhao ${ }^{1}$, Jürgen Schlegel ${ }^{4}$, Edward E. Graves ${ }^{1,2}$, Jianghong Rao ${ }^{1}$, Paul

$\triangle$ Corresponding authors: Heike E. Daldrup-Link, MD, PhD. 725 Welch Road, Rm 1665, Stanford, CA. 94305-5614 Ph: (650) 723-8996 Fax: (650) 725-8957 heiked@stanford.edu. Frederick T. Chin, PhD. 3165 Porter Drive, Rm 2129 Palo Alto, CA 94304 Ph: (650) 725-4182 Fax: (650) 618-0415 chinf@stanford.edu

(C) The author(s). This is an open access article distributed under the terms of the Creative Commons Attribution License (https://creativecommons.org/licenses/by/4.0/). See http://ivyspring.com/terms for full terms and conditions.

Received: 2019.04.10; Accepted: 2019.09.14; Published: 2019.09.17

\begin{abstract}
Despite considerable progress with our understanding of glioblastoma multiforme (GBM) and the precise delivery of radiotherapy, the prognosis for GBM patients is still unfavorable with tumor recurrence due to radioresistance being a major concern. We recently developed a cross-linked iron oxide nanoparticle conjugated to azademethylcolchicine (CLIO-ICT) to target and eradicate a subpopulation of quiescent cells, glioblastoma initiating cells (GICs), which could be a reason for radioresistance and tumor relapse. The purpose of our study was to investigate if CLIO-ICT has an additive therapeutic effect to enhance the response of GBMs to ionizing radiation.
\end{abstract}

Methods: NSGTM mice bearing human GBMs and C57BL/6] mice bearing murine GBMs received CLIO-ICT, radiation, or combination treatment. The mice underwent pre- and post-treatment magnetic resonance imaging (MRI) scans, bioluminescence imaging (BLI), and histological analysis. Tumor nanoparticle enhancement, tumor flux, microvessel density, GIC, and apoptosis markers were compared between different groups using a one-way ANOVA and two-tailed Mann-Whitney test. Additional NSGTM mice underwent survival analyses with Kaplan-Meier curves and a log rank (Mantel-Cox) test.

Results: At 2 weeks post-treatment, BLI and MRI scans revealed significant reduction in tumor size for CLIO-ICT plus radiation treated tumors compared to monotherapy or vehicle-treated tumors. Combining CLIO-ICT with radiation therapy significantly decreased microvessel density, decreased GICs, increased caspase-3 expression, and prolonged the survival of GBM-bearing mice. CLIO-ICT delivery to GBM could be monitored with MRI. and was not significantly different before and after radiation. There was no significant caspase- 3 expression in normal brain at therapeutic doses of CLIO-ICT administered.

Conclusion: Our data shows additive anti-tumor effects of CLIO-ICT nanoparticles in combination with radiotherapy. The combination therapy proposed here could potentially be a clinically translatable strategy for treating GBMs.

Key words: glioblastoma, glioblastoma initiating cells, theranostic nanoparticle, radiation therapy, imaging, ferumoxytol

\section{Introduction}

Glioblastoma multiforme (GBM) is one of the deadliest neoplasms in children and young adults, with a median survival of 12-14 months [1, 2]. The standard treatment includes maximal safe resection 
and post-operative chemo/radiotherapy [3-5]. When exposed to ionizing radiation, GBM tumor cells undergo DNA damage and tumor cell death [6]. However, glioblastoma initiating cells (GICs) pose a challenge to achieving tumor response to radiation therapy. GICs are a subpopulation of quiescent cells that can activate DNA damage repair pathways, escape the toxic effect of radiation and subsequently induce tumor recurrence [7, 8]. Increasing doses of radiation have been used to target GICs but systemic toxicity is of significant concern [9]. Therefore, finding effective combinations of radiation with other therapeutic methods to target GICs is critical for the development of more effective anti-GBM strategies. The use of nanoparticles, in particular, is attractive given the advantages of improved plasma half-life, bio-availability, and sustained controlled drug release [10].

We have recently developed and validated a treatment approach for GBM with theranostic nanoparticles (TNPs) in mouse models (Figure 1) [11]. Briefly, the TNPs are composed of FDA-approved cross-linked iron oxide nanoparticles (CLIO) and the highly effective vascular disrupting agent (VDA) azademethylcolchicine (ICT2552). CLIO and ICT2552 are linked through an MMP-14-cleavable linker to afford the MMP-14 activatable theranostic drug, CLIO-ICT [12]. Matrix metalloproteinase 14 (MMP-14) is a membrane-bound protein playing an important role in the stemness of aggressive cancers, including GBM [13-15], which makes it an appealing therapeutic target for GICs.
Here, we propose a new strategy of combining theranostic nanoparticles with radiation therapy (RT) for improved treatment of glioblastomas. The theranostic nanoparticles disrupt the blood brain barrier in the tumor tissue through MMP-14 mediated activation of the VDA prodrug. MMP-14 activation releases the vascular disrupting agent, ICT, which initiates a positive feedback loop of nanoparticle uptake and subsequent drug release. BBB breakdown releases the therapeutic drug ICT to the perivascular niche, where GICs are preferentially located. Since MMP-14 is abundant in GBM and not normal brain [13, 16-19], CLIO-ICT provides highly specific destruction of tumor vasculature and highly specific elimination of GBM tumor cells and GICs residing in the perivascular niche. Additionally, the CLIO nanocarrier allows for in vivo drug tracking with magnetic resonance imaging (MRI) [11, 12].

Thus, we hypothesize that CLIO-ICT, by targeting GICs, will have additive anti-GBM effects in combination with radiation. In addition, we postulate that CLIO-ICT will improve the efficacy of radiotherapy with marginal toxic effects to the normal brain and visceral organs.

\section{Methods}

Chemicals and antibodies. The following antibodies were used: MMP-14 (Santa Cruz), CD31 (Abcam), Desmin (Abcam), CD15 (Abcam), and cleaved caspase-3 (Cell Signaling Technology). The following chemicals were used: Ferumoxytol (AMAG Pharmaceuticals). ICT and TNP were synthesized and characterized according to a previous protocol [11].

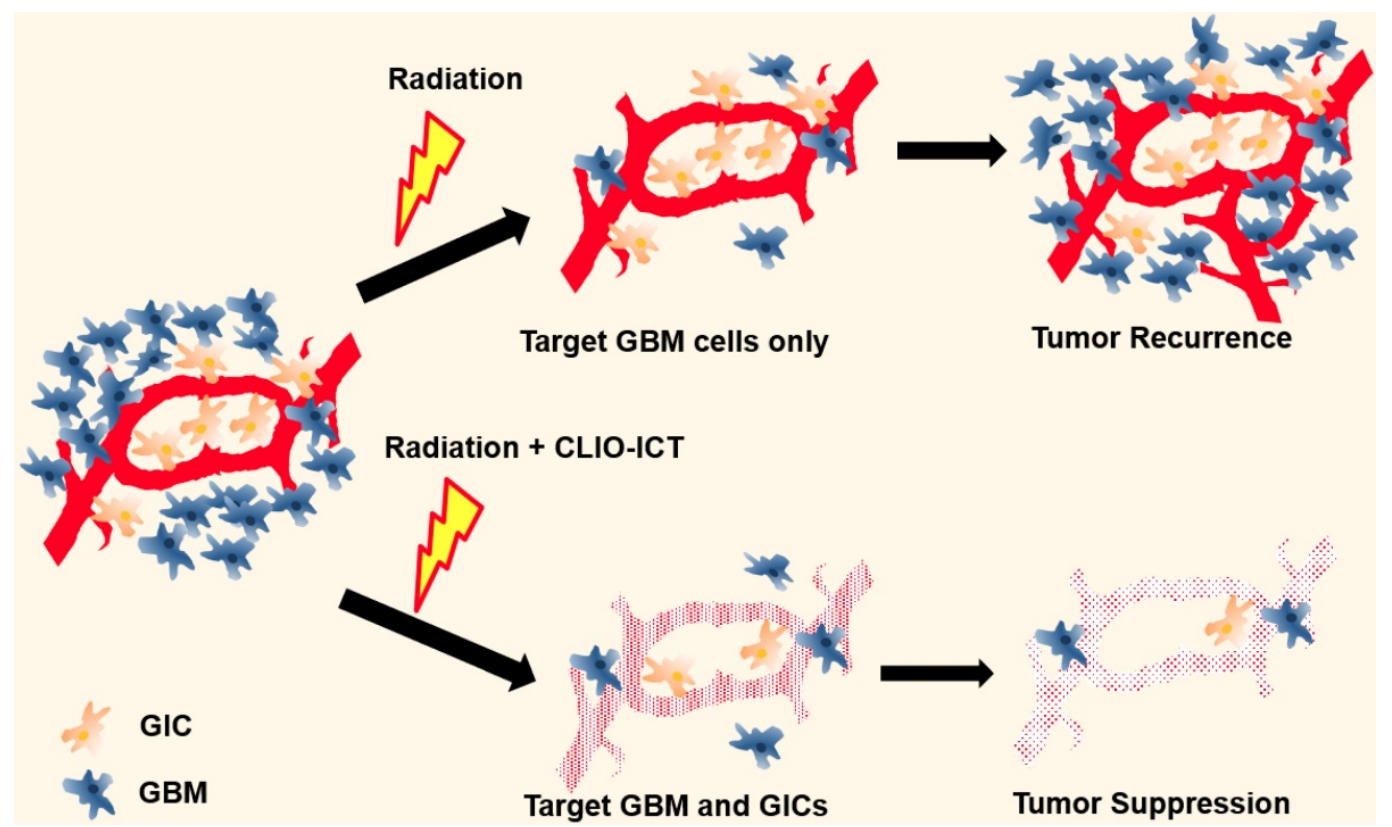

Figure 1: Illustration of additive effects of CLIO-ICT on radiotherapy. Radiotherapy targets glioblastoma cells but has a limited effect on glioblastoma initiating cells (GICs). GICs are often radioresistant and important for tumor recurrence. CLIO-ICT targets the vascular niche and indiscriminately induces apoptosis of both highly proliferated GBM cells and quiescent GICs. Therefore, CLIO-ICT could potentially overcome radioresistance and increase the efficacy of radiotherapy in GBMs. 
Cell culture. A patient-derived GBM cell line, GBM39, and a murine GBM cell line, CT-2A, were used in vitro and in vivo. The cell lines GBM39 and CT-2A were kindly provided by Dr. Sanjiv Sam Gambhir and Dr. Samuel Cheshier (Stanford University). CT-2A cells were grown in DMEM (Life Technologies) containing $10 \%$ FBS and $1 \%$ Penicillin/Streptomycin (Life Technologies). GBM39 were cultured as floating cellular spheres as previously described [11]. All cell lines were authenticated and confirmed to be mycoplasma negative using the MycoAlert Mycoplasma Activity Kit (Lonza). All cell lines used were from early passages and were transfected with firefly luciferase enzyme to enable monitoring of tumor growth using bioluminescence imaging.

Animal models. To generate orthotopic GBM xenografts, 300,000 tumor cells were injected stereotactically into the striatum of 8-week-old female NOD scid gamma (NSG ${ }^{\mathrm{TM}}$ ) and C57BL/6J mice, using the following coordinates: $2 \mathrm{~mm}$ posterior to the bregma, $2 \mathrm{~mm}$ lateral to the midline, and $3 \mathrm{~mm}$ deep with respect to the surface of the skull. All animal maintenance, handling, surveillance, and experimentation were performed in accordance with and approval from the Stanford University Administrative Panel on Laboratory Animal Care (Protocol 24965).

MMP-14 expression in patient tumor and murine tissues. To confirm that human GBMs have greater MMP-14 expression than normal brain to activate CLIO-ICT, we evaluated the expression of MMP-14 in GBM specimen from 5 chemotherapy-naïve human patients (kindly provided by Dr. Jürgen Schlegel, Neuropathology, Technical University of Munich, Germany) as well as murine GBM specimen from GBM39-inoculated mice using immunohistochemistry (IHC). To estimate CLIO-ICT activation in normal organs, we additionally evaluated MMP-14 expression in murine lung, liver, heart, kidney, and spleen obtained from three C57BL/6J mice. IHC for MMP-14 was performed using the micro-polymer-IHC detection kit (Abcam) following the manufacturer's instructions. Representative images were captured using a Zeiss AxioImager Widefield fluorescence microscope with a 20X objective for whole-slide imaging (evaluated according to Immunoreactivity Score (IRS) system, using the following scores for percentage of positive cells: Negative (0), $\leq 10 \%(1), \geq 11 \%$ and $\leq 50 \%(2), \geq$ $51 \%$ and $\leq 80 \%$ (3), $\geq 81 \%$ (4).

Dose response studies. Fifteen $\mathrm{NSG}^{\mathrm{TM}}$ mice and fifteen C57BL/6J mice were randomized in five groups (3 mice/group) receiving CLIO-ICT intravenously at different doses $(0,1,2,5$ and 10 $\mathrm{mg} / \mathrm{kg} \mathrm{ICT} ; 0,4.25,8.5,17,34 \mathrm{mg} / \mathrm{kg} \mathrm{Fe})$. PBS was administered as vehicle in NSG ${ }^{\mathrm{TM}}$ and C57BL/6J mice without CLIO-ICT treatment. Bioluminescence imaging (BLI) was used to determine the anti-tumor effects of CLIO-ICT at various doses. The dose at which we observed maximum inhibition of tumor growth was considered as the optimum therapeutic dose in vivo.

Monitoring tumor growth with BLI. Tumor formation of the animals were scored by BLI (twice per week after inoculation). Luminescent imaging was performed on an IVIS Spectrum (Caliper Life Science) and quantified using Living Image 4.0 software. D-Luciferin (firefly) potassium salt solution (Biosynth) was prepared $(15 \mathrm{mg} / \mathrm{mL}$ in PBS) and injected intraperitoneally $(90 \mathrm{mg} / \mathrm{kg}, \sim 150 \mu \mathrm{L})$. Total flux (photons per second) values were obtained by imaging repeatedly until peak radiance was achieved and quantified with Living Image 4.0 software. BLI was repeated at the end of the treatment.

Toxicity studies. Toxicity was evaluated by hematoxylin and eosin (H\&E) staining, aspartate aminotransferase (AST), alanine aminotransferase (ALT), and creatinine activities. An additional set of thirty non-tumor bearing C57BL/6J mice (6 mice/group) received intravenous injections of PBS, CLIO-ICT $(10 \mathrm{mg} / \mathrm{kg}$ ICT; $34 \mathrm{mg} / \mathrm{kg} \mathrm{Fe})$, CLIO-ICT (70 mg/kg ICT; $238 \mathrm{mg} / \mathrm{kg}$ Fe), colchicine $(2 \mathrm{mg} / \mathrm{kg})$, or colchicine $(10 \mathrm{mg} / \mathrm{kg})$. Given that our theranostic drug is activated in tissue by MMP-14 to release the vasculature disruptive agent, azademethylcolchicine (a colchicine derivative), we used equimolar doses of colchicine, assuming that all CLIO-ICT is cleaved in target tissues. Colchicine $2 \mathrm{mg} / \mathrm{kg}$ and $10 \mathrm{mg} / \mathrm{kg}$ corresponds to $10 \mathrm{mg} / \mathrm{kg}$ ICT and $70 \mathrm{mg} / \mathrm{kg}$ ICT, respectively. After day 5 of treatment, blood was collected into heparinized tubes to separate the plasma and stored in $-80{ }^{\circ} \mathrm{C}$. Liver toxicity was assayed using ALT/SGPT Activity Kit (BioVision K752-100) and AST/SGOT Activity Assay Kit (BioVision K753-100) following the manufacturer's instructions. Renal toxicity was assayed using Creatinine (CRE) Assay Kit (MyBioSource MBS2540563) following the manufacturer's instructions. Several organs (liver, heart, kidney, spleen, bone marrow, and brain) were harvested from all animals and fixed in 10\% neutral buffered formalin for $24 \mathrm{~h}$, followed by $70 \%$ ethanol at room temperature for $24 \mathrm{~h}$. Organs were then embedded in paraffin for $3 \mathrm{~h}$ at $67^{\circ} \mathrm{C}$. Coronal sections ( $5 \mu \mathrm{m}$ thick) were stained with hematoxylin and eosin, and images were acquired with Eclipse E800 camera (Nikon, USA) [11].

Efficacy of CLIO-ICT or/and radiation combination therapies. Twenty-eight $\mathrm{NSG}^{\mathrm{TM}}$ mice 
and twenty-four C57BL/6J mice with GBM were randomized into five different treatment groups $(n=6$ mice/group), which were treated as follows: Group 1: CLIO-ICT treatment $(10 \mathrm{mg} / \mathrm{kg}$ ICT, $34 \mathrm{mg} / \mathrm{kg} \mathrm{Fe})$, Group 2: radiation therapy (10 Gy), Group 3: CLIO-ICT + radiation therapy (same doses as in groups 1 and 2), Group 4: phosphate buffered saline (PBS), and Group 5: temozolomide (TMZ, $33 \mathrm{mg} / \mathrm{kg}$ ) + radiation therapy. In group 5, two mice died of their brain cancer before completion of the experiment. Once tumor growth had been confirmed by BLI, the above-mentioned therapeutic regimen was administered on day 0 and day 7. Radiation therapy preceded intravenous injection of the therapeutic nanoparticles by 3 hours.

Computed tomography (CT) and radiotherapy. Treatment planning and precise delivery of radiation was performed using a PXi X-Rad SmART cabinet irradiator (Precision X-Ray Inc., North Branford, CT). The system has the X-ray tube and the detector plate mounted on $\mathrm{U}$ shape gantry that rotates $360^{\circ}$ in $\mathrm{X}$ and $Y$ plane around the animal stage. The animal stage is supplied by a nose cone delivering isoflurane anesthesia and can move $\pm 10 \mathrm{~cm}$ in the $X, Y$ directions and $\pm 15 \mathrm{~cm}$ in $Z$ direction. CT images were acquired to facilitate treatment planning using a beam energy of $40 \mathrm{kVp}$, a beam filter of $2 \mathrm{~mm} \mathrm{Al}$ and a voxel size of 0.2 or $0.1 \mathrm{~mm}$. Therapeutic irradiations were performed using an X-ray energy of $225 \mathrm{kVp}$ and a current of $13 \mathrm{~mA}$ producing a dose rate of 300 $\mathrm{mGy} / \mathrm{min}$ at the isocenter. A single dose of $10 \mathrm{~Gy}$ was delivered using a $10 \mathrm{~mm}$ collimator. The treatment planning was performed with an open source RT image software package, version 3.13.1 running on IDL version 8.5.1, where the CT images were used as a reference to decide the beam angle and collimator size [21, 22].

Magnetic resonance imaging (MRI). We evaluated tumor delivery of CLIO-ICT in GBM-bearing mice using MRI. GBM39-bearing mice underwent MRI before, 24 hours after, and 14 days after treatment with PBS, radiation, CLIO-ICT, radiation + CLIO-ICT. MRI studies of GBM-bearing mice were performed on a 7T MR scanner (Bruker Biospin, Billerica, MA), using an appropriate surface coil and the following pulse sequences with a field of view of $2 \mathrm{~cm} \times 2 \mathrm{~cm}$ and a slice thickness of $0.5 \mathrm{~mm}$ : $\mathrm{T}_{2}$-weighted fast spin echo (FSE): repetition time (TR): $3476 \mathrm{~ms}$, echo time (TE): $33 \mathrm{~ms}$, flip angle a: $90^{\circ}$ and $\mathrm{T}_{2}$ multi-slice multi-echo (MSME): TR: $2964 \mathrm{~ms}$, TE: 8, 16, $24,32,40,48,56,64,72,80,88$ and $96 \mathrm{~ms}$, a: $90^{\circ}$. $\mathrm{T}_{2}$ relaxation times of the tumors were calculated as a quantitative measure of tumor contrast enhancement.

Immunofluorescence. Mice in groups 1-5 were euthanized after the last imaging procedure for immunofluorescence. Brain tissues were also fixed in $4 \%$ paraformaldehyde at $4^{\circ} \mathrm{C}$ overnight and later immersed in $30 \%$ sucrose for 2 days. Afterwards, brains were then embedded in OCT and stored in -80 ${ }^{\circ} \mathrm{C}$. Coronal sections ( $5 \mu \mathrm{m}$ thick) were mounted on superfrost slides, rinsed with PBS and permeabilized with $0.1 \%$ Triton X-100 made in PBS solution for 15 min. Subsequently, cells were blocked for $2 \mathrm{~h}$ and stained with primary antibodies overnight to determine GIC populations or to evaluate apoptosis upon treatment with radiation and/or CLIO-ICT. The following dilutions were used: CD31 (1:20, Abcam), desmin (1:50, Abcam), CD15 (1:200, Abcam) and cleaved caspase-3 (1:300, Cell Signaling Technology). Nuclei were counterstained with DAPI. Single endothelial cells or clusters of endothelial cells that were positive for CD-31 and desmin were considered as a vessel. Immunofluorescence images were acquired with a Leica SP8 confocal microscope (20X) using Leica AF software. Three "hot spots" from each slide were identified and photographed. The total number and positive number of cells were counted with Image-J in a blindfolded manner and were estimated as a mean \pm SD in three different fields from three independent experiments.

Survival studies. Mice with GBM39 tumors were treated with CLIO-ICT $(10 \mathrm{mg} / \mathrm{kg}$ ICT, 34 $\mathrm{mg} / \mathrm{kg} \mathrm{Fe}$ ), radiation (10 Gy), CLIO-ICT + radiation, or PBS using the same protocols as above (6 mice/group). Animal survival was evaluated from the first day of treatment until death. Body weight was measured twice a week. To avoid animal suffering, animals were euthanized when meeting predefined criteria, including loss of ability to ambulate, labored respiration, or inability to drink or feed.

Prussian blue staining. The biodistribution of CLIO-ICT was investigated by Prussian blue staining. A set of nine mice with GBM39 tumors were treated with PBS, CLIO-ICT (10 mg/ kg ICT, $34 \mathrm{mg} / \mathrm{kg} \mathrm{Fe})$, or CLIO-ICT $(10 \mathrm{mg} / \mathrm{kg} \mathrm{ICT,} 34 \mathrm{mg} / \mathrm{kg} \mathrm{Fe})+$ radiation (10 Gy) (3 mice/group). At 72 hours post-treatment, the mice were perfused with PBS and the tumor and visceral organs were harvested. The presence of iron in these specimens was investigated with Prussian blue staining using Iron Stain Kit (Sigma) according to the manufacturer's instructions. Briefly, the tissue slides were deparaffinized and hydrated with deionized water. Subsequently, the slides were placed in working iron stain solution for $10 \mathrm{~min}$. After rinsing with deionized water, the tissue was stained with Nuclear Fast Red solution (Sigma) for $5 \mathrm{~min}$. Lastly, the tissue was rapidly dehydrated with alcohol and xylene and mounted for imaging. Images were acquired with Eclipse E800 camera (Nikon, USA). 
Three "hot spots" from each slide were identified and were photographed (40X). The blue dots were counted with Image-J in a blindfolded manner and were estimated as a total of number of prussian blue positive cells per 100 cells (\%) \pm SD in three different fields from three independent experiments.

Intravital microscopy (IVM). A calvarial defect with a 5-mm diameter was created in an 8-week-old NOD scid gamma (NSG ${ }^{\mathrm{TM}}$ ) mouse under anesthesia with $2 \%$ isoflurane delivered in $100 \%$ oxygen. To generate orthotopic GBM xenografts, GBM39 cells (Total $=300,000)$ were injected stereotactically into the striatum as described above. Prior to implantation, the cells were labeled with CellbriteTM Red (Biotium 30023) according to the manufacturer's instructions. The defect was covered with a cover glass slide and an MR-compatible cranial window chamber to enable real time imaging of tumor cells and tumor cell vasculature. For imaging, the head was immobilized using a stereotactic frame coupled with ear bars (Kopf Instruments, Tujunga, CA). Images were acquired before and after treatment with FITC-labeled CLIO-ICT or CLIO using a microscope (IV-100; Olympus, Tokyo, Japan) with Olympus UplanFL objectives and Olympus FluoView FV300 software. Time per pixel was set to 8-12.5 $\mu \mathrm{sec}$, and voltage was set to 500-600 V. Images were analyzed with ImageJ software (National Institutes of Health; Bethesda, MD).

Statistical analysis. The presence of statistical significance in the data set was determined using one-way ANOVA test. A nonparametric two-tailed Mann-Whitney test was used to compare two groups, unless otherwise specified. Results were presented as mean \pm SD. Kaplan-Meier survival curves were compared using the log-rank (Mantel-Cox) test. The level of significance was set at $p<0.05$, as compared with the control group. Statistical analyses were carried out with Prism 6.0 software (GraphPad).

\section{Results}

Development of theranostic nanoparticles. As previously described, we developed ICT, a peptide-conjugate of the VDA azademethylcolchicine (ICT2552), which is cleaved by MMP-14 to release and activate the therapeutic drug $[12,23]$. To overcome potential limited tumor retention and systemic toxicity of this small molecular therapeutic drug, we attached the ICT prodrug to the cross-linked iron oxide nanoparticle compound, ferumoxytol [12]. CLIO-ICT nanoparticles have a diameter of $21 \pm 3 \mathrm{~nm}$ (Dynamic Light Scattering) and a zeta potential of $21 \pm 7 \mathrm{meV}$ (Laser Doppler Electrophoresis). The number of ICT molecules per iron oxide nanoparticle was determined to be on average 4.7 , based on the attached fluorescein absorption in samples with known nanoparticle concentration [12].

Expression of MMP-14 in tumor and normal organs. As a prerequisite for CLIO-ICT activation in GBMs, we first investigated whether human GBMs express MMP-14. IHC confirmed that MMP-14 protein levels in tumor regions of all GBM patient specimens were significantly $(\mathrm{p}<0.001)$ higher compared to normal brain regions (Figure 2A). We confirmed MMP-14 expression in murine GBMs (Figure 2B) and demonstrated CLIO-ICT dose-dependent inhibition of tumor growth in both xenograft and syngeneic GBM models. With a dose of ICT $(10 \mathrm{mg} / \mathrm{kg})$ and $\mathrm{Fe}(34 \mathrm{mg} / \mathrm{kg})$, CLIO-ICT showed the maximum anti-GBM effect in both human (Figure 2C \& 2E) and murine GBMs (Figure 2D \& 2F) - thus, this optimal dose was used for subsequent combination therapies with radiation. To exclude toxicity in normal organs, we screened tissue specimen of the brain and visceral organs for MMP-14 expression (Figure 3A). MMP-14 expression in the tumor was twice as high as lung and liver and 3-4 times higher than in the kidney, heart, and spleen (Figure 3A \& 3B).

Toxicity studies. Liver toxicity markers (AST and ALT in plasma) were significantly lower for mice treated with CLIO-ICT compared with mice treated with free colchicine (Figure 3D \& Figure 3E, p < 0.0001). Creatinine levels in plasma demonstrated a similar pattern at the therapeutic dose of CLIO-ICT (ICT $10 \mathrm{mg} / \mathrm{kg}$ and Fe $34 \mathrm{mg} / \mathrm{kg}$ (Figure 3F, p < 0.01). However, excessive doses of CLIO-ICT caused significantly increased creatinine compared to colchicine treated mice (Figure 3D, $\mathrm{p}<0.0001$ ). H\&E staining further demonstrated signs of necrosis in brain, heart, kidney, liver, spleen, and bone marrow in colchicine treated animals $(2 \mathrm{mg} / \mathrm{kg}$, Figure $3 \mathrm{C})$. By comparison, organs from CLIO-ICT-treated animals showed no signs of necrosis (ICT $10 \mathrm{mg} / \mathrm{kg}$ and Fe 34 $\mathrm{mg} / \mathrm{kg}$, Figure 3C).

CLIO-ICT biodistribution. To evaluate CLIO-ICT biodistribution, mice were treated with PBS, CLIO-ICT, or radiation + CLIO-ICT and then perfused with PBS $72 \mathrm{hrs}$ later. Tissues were harvested and stained with Prussian blue to examine the extent of iron uptake in the tumor, brain and visceral organs (Supplementary Figure 1). Compared to PBS treated controls, we found significantly higher iron content in CLIO-ICT treated tumors ( $p<0.001)$. In each treatment group, GBMs demonstrated significantly higher iron uptake than the normal brain regardless of whether the mice were treated with CLIO-ICT alone $(p<0.001)$ or in combination with radiation $(p<0.001)$. The degree of iron uptake in the tumor between the monotherapy and combined 


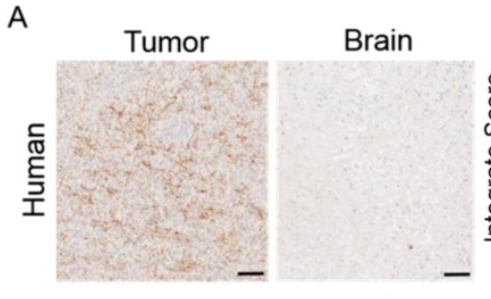

C

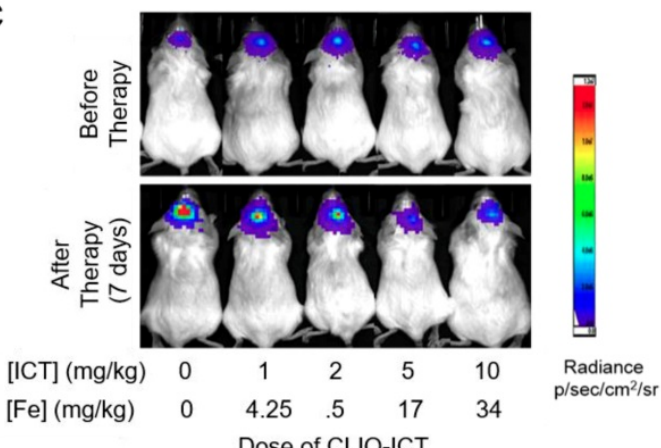

E

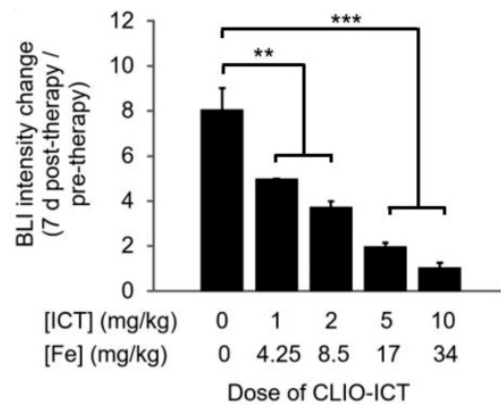

B
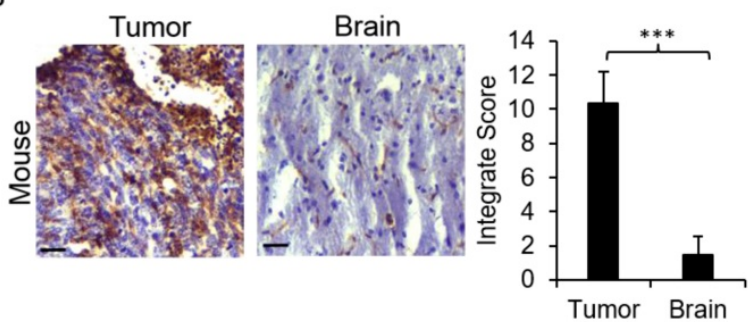

D

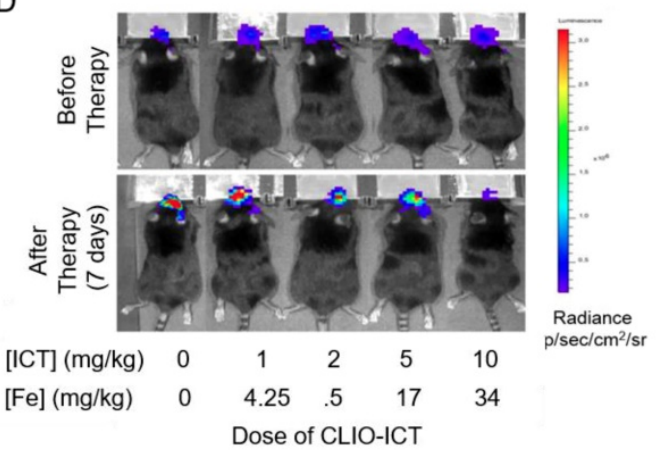

F

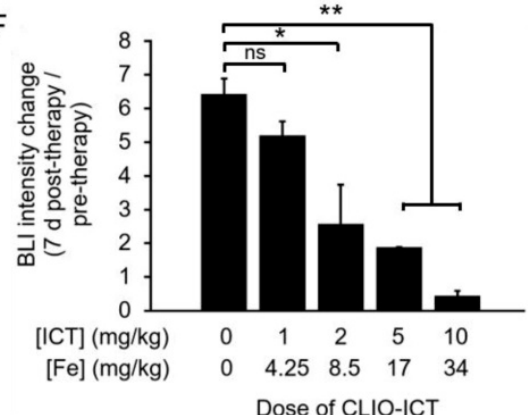

Figure 2: Dose response studies of CLIO-ICT in human and murine GBMs. (A) MMP-14 immunohistochemistry staining of human GBM and normal human brain specimen (scale bar, $100 \mu \mathrm{m}$ ). Immunohistochemistry results were evaluated by a semi-quantitative approach. The score for intensity is $0,1+, 2+, 3+$. The percentage of cells is scored : Negative (0), $\leq 10 \%(1), \geq 11 \%$ and $\leq 50 \%(2), \geq 51 \%$ and $\leq 80 \%(3), \geq 81 \%(4)$. The formula for integrate score: the score for the intensity $\times$ the score for percentage of positive cells. (B) MMP-14 staining in murine CT-2A GBM tumors and normal murine brain specimen (scale bar, $40 \mu \mathrm{m}$ ). Immunohistochemistry results were evaluated by a semi-quantitative approach as mentioned above. (C-F) Dose-dependent effects of CLIO-ICT: Bioluminescent in vivo images of human GBM39 tumors in NSG ${ }^{\mathrm{TM}}$ mice (C) and murine CT-2A tumors in C57BL/6] mice (D). Ratio of bioluminescence radiance before and after therapy for human GBM39 tumors (E) and murine CT-2A tumors (F). Results are represented as the mean \pm SD $\left(n=3\right.$ for NSG ${ }^{\mathrm{TM}}$ mice, $\mathrm{n}=2$ for C57BL/6J mice), ${ }^{*} \mathrm{p}<0.05$, ** $\mathrm{p}<0.01$, *** $\mathrm{p}<0.001$, ns $=$ not significant.

therapy groups was not significantly different (Supplementary Figure 1) demonstrating consistent delivery of the TNPs to the tumor.

Compared to PBS treated controls, there was no significant iron uptake in the heart, lung, or kidney in mice treated with CLIO-ICT. However, we found significantly higher iron content in the liver of mice treated with CLIO-ICT compared to mice treated with PBS (Supplementary Figure 1). Mice have an intrinsically high iron content in the spleen as one of the spleen's chief roles is to filter blood and recycle iron from aging blood cells. The red pulp of the spleen is also home to mononuclear phagocytes (i.e., macrophages, dendritic cells, and monocytes) which are known to consume iron oxide particles [24]. Our results showed that the iron content in the spleen of mice treated CLIO-ICT and PBS was not significantly different. The radiation therapy was collimated to the brain only, therefore, we neither expected nor observed a difference in the iron staining of visceral organs between the CLIO-ICT and CLIO-ICT plus radiation treatment groups.

Preliminary intravital microscopy studies in NSG $^{\text {TM }}$ mice with orthotopic GBM3 xenografts showed that intravenously administered CLIO-ICT disrupted the BBB and accumulated in the extravascular space of the tumor tissue (Supplementary Figure 2, green fluorescence). Since MMP-14 is overexpressed on both tumor endothelial cells and tumor cells, CLIO-ICT is activated and released in the tumor vasculature, leading to efficient breakdown of the blood brain barrier (BBB). After intravenous injection of FITC-conjugated CLIO-ICT, the FITC signal in the tumor interstitium was significantly more intense compared to the FITC signal in the tumor interstitium after intravenous injection of CLIO only (without attached vascular disrupting agent). This suggests that CLIO-ICT tumor accumulation is markedly increased due to VDA-mediated vascular disruption. These findings 
correlated with results of the MRI studies: At $24 \mathrm{~h}$ after intravenous injection of CLIO-ICT treatment, tumor T2 relaxation times were significantly shorter compared to CLIO treated controls $(p<0.05)$. In addition, histology studies at later time points demonstrated tumor vessel disruption and reduced vessel density (quantified as reduced CD31 vessel staining) in CLIO-ICT treated groups compared to CLIO treated groups. CLIO treatment only did not have any effect on tumor vessels, CD31 vessel staining of CLIO treated tumors was not significantly different compared to PBS treated controls (data not shown).

Additive effects of CLIO-ICT with radiation therapy. Next, we assessed whether CLIO-ICT improves the efficacy of radiotherapy for anti-GBM treatment. Two weeks after initiation of treatment, BLI scans revealed significant reduction in tumor size for radiation plus CLIO-ICT-treated GBM39 tumors (Figure 4A \& 4B, p < 0.001) and CT-2A tumors (Figure $4 \mathrm{C} \& 4 \mathrm{D}, \mathrm{p}<0.001)$ compared to monotherapy or vehicle-treated tumors. Additional mice were treated with the standard of care therapy (TMZ + radiation), which inhibited tumor growth compared to untreated controls. However, the tumor volume of mice treated with TMZ + radiation did not change significantly compared to baseline scans before start of therapy (Supplementary Figure 3). MR scans further demonstrated CLIO-ICT delivery into tumors by a negative (dark) signal enhancement on T2-weighted MR images (Figure 5A \& 5B). T2 relaxation times of tumors treated with CLIO-ICT or radiation plus CLIO-ICT were two-fold lower compared to radiation and PBS treated tumors. The T2 relaxation times of GBMs after treatment with CLIO-ICT and CLIO-ICT plus radiation was not significantly different (see Figure 5B, $p>0.05$ ), suggesting that CLIO-ICT delivery to tumors did not change significantly with added radiation. Likewise, T2 relaxation times of normal brain regions were not significantly different between mice treated with CLIO-ICT, radiation plus CLIO-ICT, or PBS suggesting that CLIO-ICT did not accumulate in normal brain before or after radiation therapy (Figure 5C).

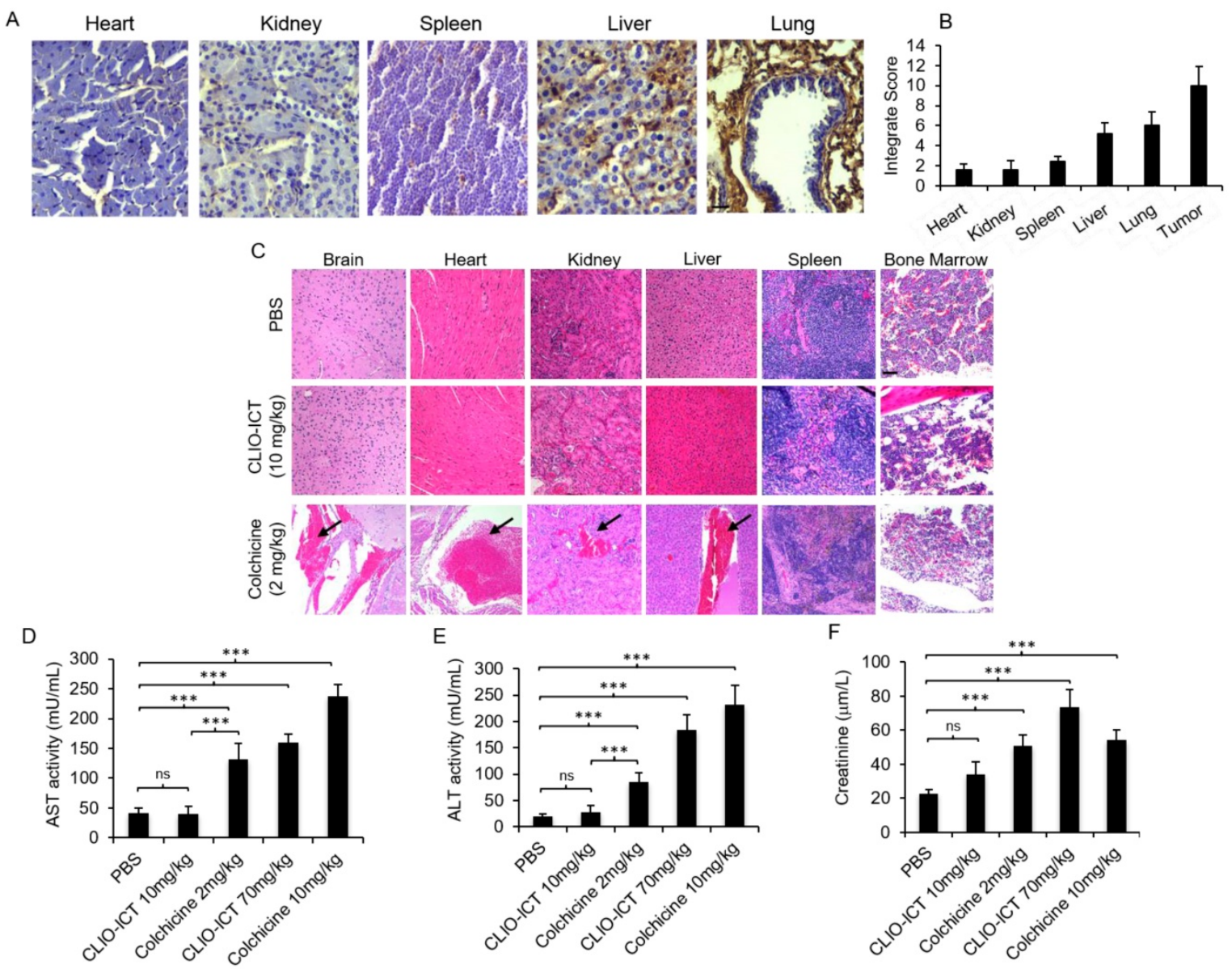

Figure 3: Safety profile of CLIO-ICT. (A) MMP-14 immunohistochemistry staining of different organs (heart, kidney, spleen, liver and lung) in C57BL/6J mice (20X magnification). (B) Immunohistochemistry results were evaluated by a semi-quantitative approach as previously described. (C) Representative H\&E images show significant necrosis in different organs in colchicine $(2 \mathrm{mg} / \mathrm{kg})$ treated mice $(\mathrm{n}=6)(10 \mathrm{X}$ magnification). Black arrows indicate necrosis. Organs from CLIO-ICT $(10 \mathrm{mg} / \mathrm{kg} \mathrm{ICT})$ treated mice appear normal and healthy. The graphs show liver toxicity markers, including (D) AST activity, (E) ALT activity, and (F) creatinine concentration in mice treated with PBS, 10 $\mathrm{mg} / \mathrm{kg}$ or $70 \mathrm{mg} / \mathrm{kg}$ CLIO-ICT, and $2 \mathrm{mg} / \mathrm{kg}$ or $10 \mathrm{mg} / \mathrm{kg}$ colchicine. Results are represented as mean $\pm \mathrm{SD}$. ${ }^{*} \mathrm{p}<0.05$, $* * \mathrm{p}<0.01, * * * \mathrm{p}<0.001$, ns $=$ not significant. 
A

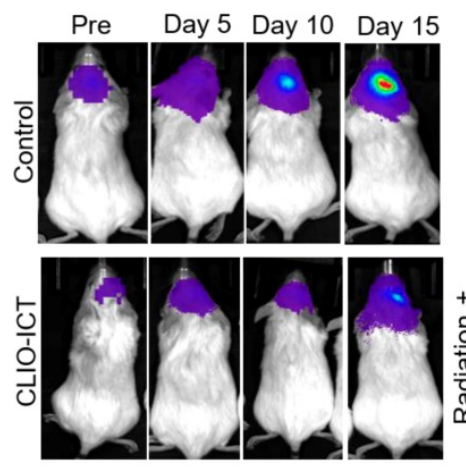

C
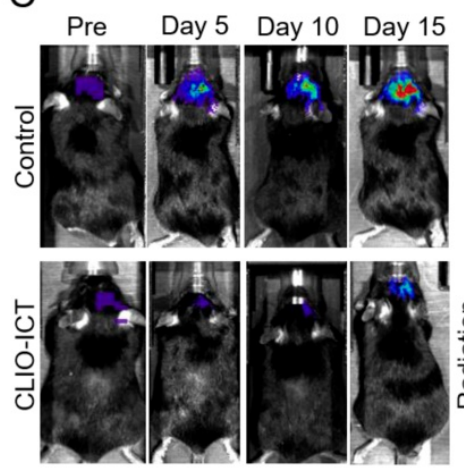
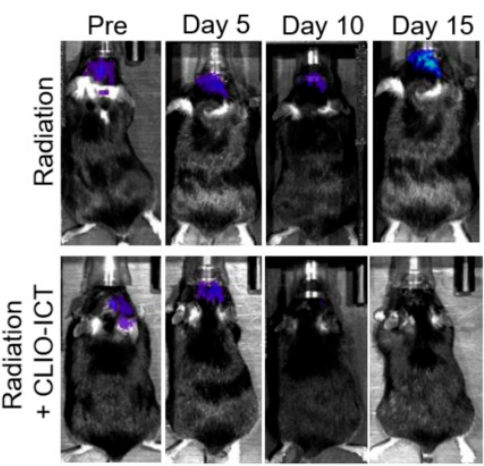

B
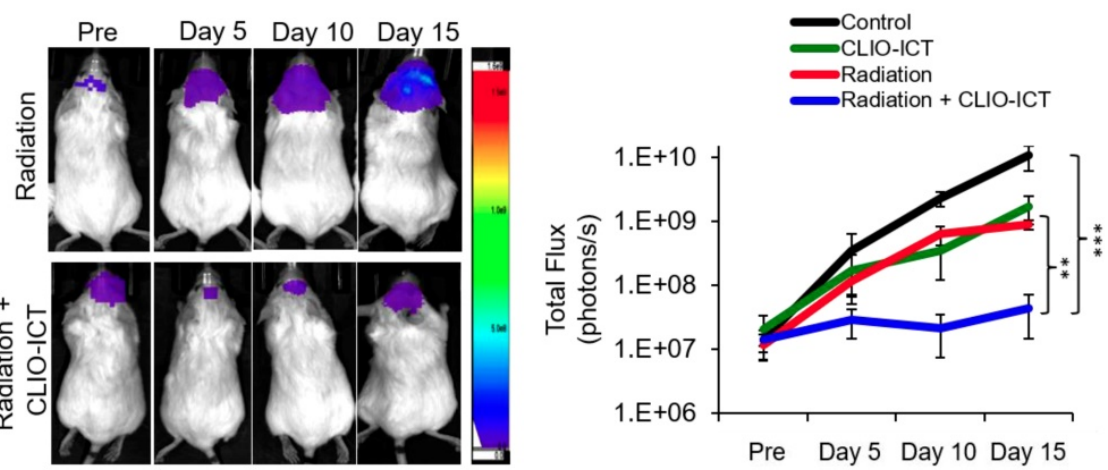

D

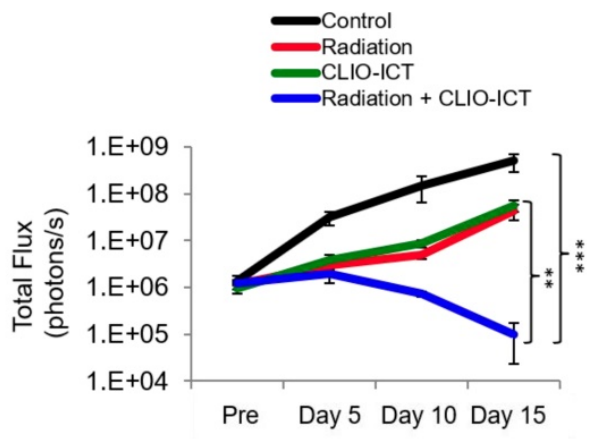

Figure 4: Efficacy studies of radiation with/or CLIO-ICT therapy in vivo. Bioluminescence images of (A) NSGTM mice with orthotopic luciferase-expressing GBM39 tumors or (C) C57BL/6] mice with orthotopic GFP-luciferase-expressing CT-2A tumors. Mice were treated with CLIO-ICT (10 mg/kg ICT, $34 \mathrm{mg} / \mathrm{kg}$ Fe) and/or radiation (10 Gy). PBS treatment served as control. Corresponding total flux of tumors before and during treatment are shown for (B) NSGTM and (D) C57BL/6J mice. All mice were treated on Day 0 and again on Day 7. Results are represented as mean \pm SD from six animals, $* p<0.05, * * p<0.01$, *** $p<0.001$, ns $=$ not significant. Pre $=$ pre-therapy.

A

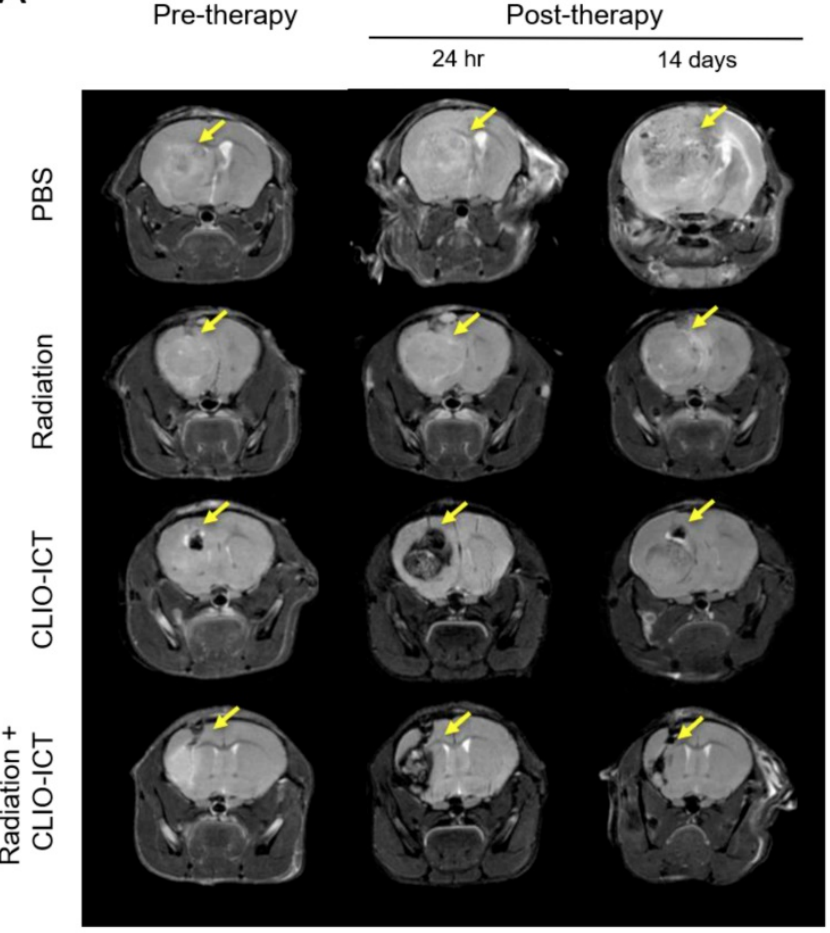

B - Pre-therapy $\quad$ - Post-therapy $(24 \mathrm{hr})$

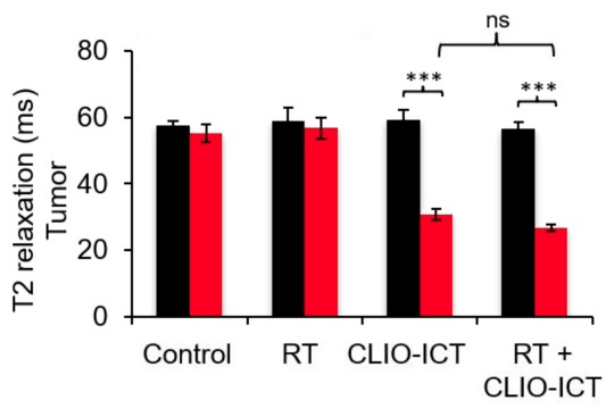

C

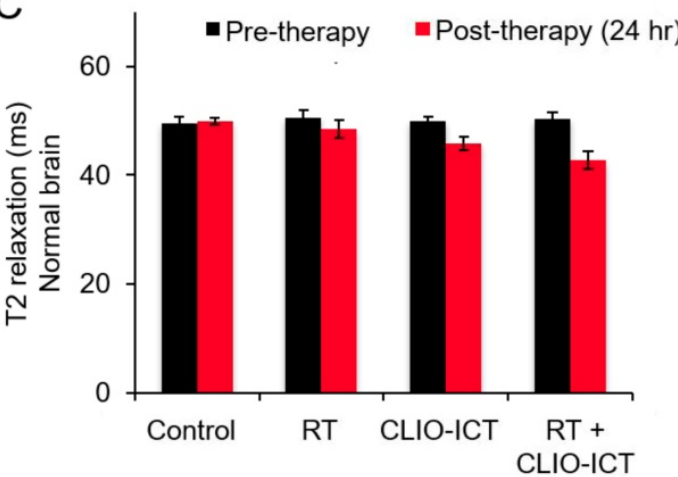

Figure 5: MR scans after radiation with/or CLIO-ICT therapy. (A) Representative coronal T2-weighted MR images of the brain of NSGTM mice with GBM39 tumors before and after CLIO-ICT (10 mg/kg ICT, $34 \mathrm{mg} / \mathrm{kg}$ Fe) and/or radiation (10 Gy) treatment ( $24 \mathrm{hr}$ and $14 \mathrm{~d}$ ). PBS treatment served as control. Theranostic nanoparticle delivery is demonstrated by hypointense (negative/dark) tumor enhancement in CLIO-ICT and CLIO-ICT + radiation treated animals. Yellow arrows indicate the site of tumor growth. (B) Corresponding T2 relaxation times of tumors before and $24 \mathrm{hr}$ after therapy. Note significant shortening of T2 relaxation times of tumors after administration of CLIO-ICT. (C) T2 relaxation times of the normal brain before and $24 \mathrm{hr}$ after therapy. Data represent mean \pm SD from six animals, $* \mathrm{p}<0.05$, $* * \mathrm{p}<0.01$, $* * * \mathrm{p}<0.001$, ns $=$ not significant. 
A
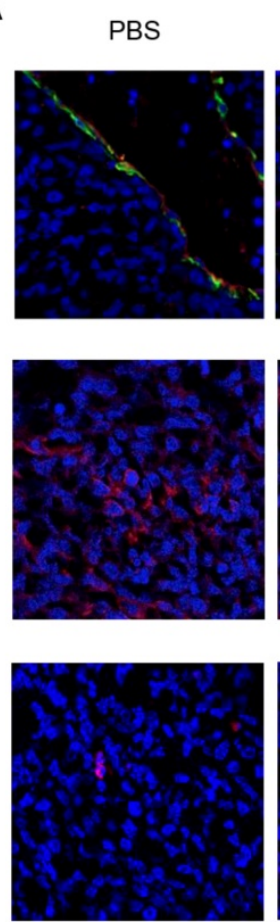

Radiation
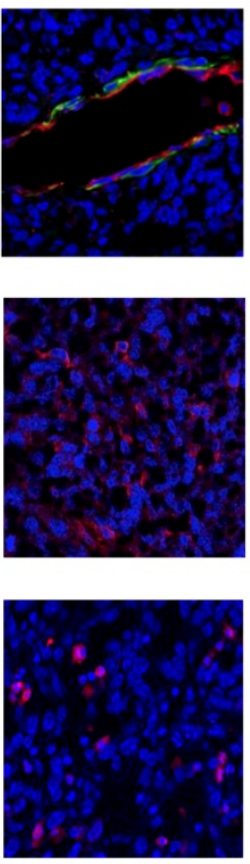

CLIO-ICT
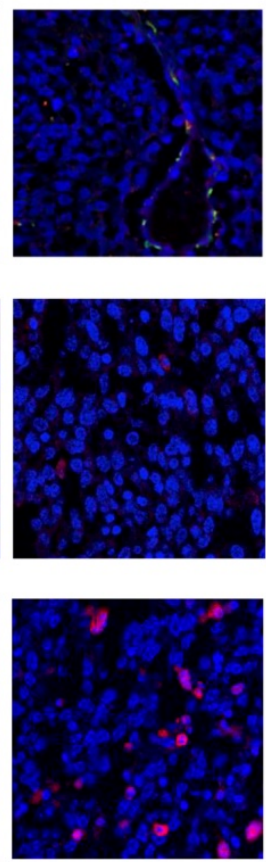

Radiation + CLIO-ICT
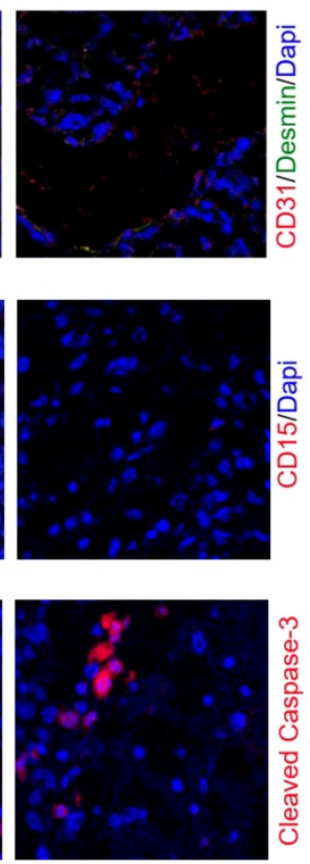
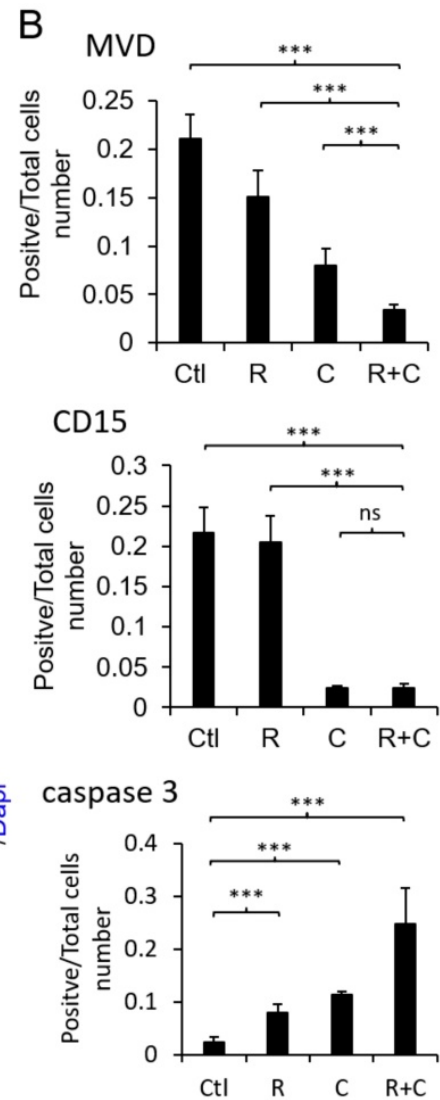

Figure 6: Effect of mono- or combined-therapy on GBM vasculature and GIC population. (A) Representative confocal images depicting the effect of mono and combination therapy on tumor vasculature, GICs, and GBM cell death. GBM vasculature is outlined by staining with endothelial cell marker-CD31 and pericyte marker-desmin; GICs are identified using CD15, and GBM cell death is identified with cleaved caspase-3 (20X magnification). (B) Graphs show quantification of tumor microvessel density (MVD), CD 15, and cleaved caspase-3 area. Results are represented as mean \pm SD from six animals, ${ }^{*} \mathrm{P}<0.05$, $* * \mathrm{P}<0.01$, *** $\mathrm{P}<0.001$, ns $=$ not significant. Tissue sections are from mice that were sacrificed 15 days post-treatment. $C t I=P B S$ control, $R=$ radiation, $C=C L I O-I C T, R+C=$ radiation $+C L I O-I C T$.

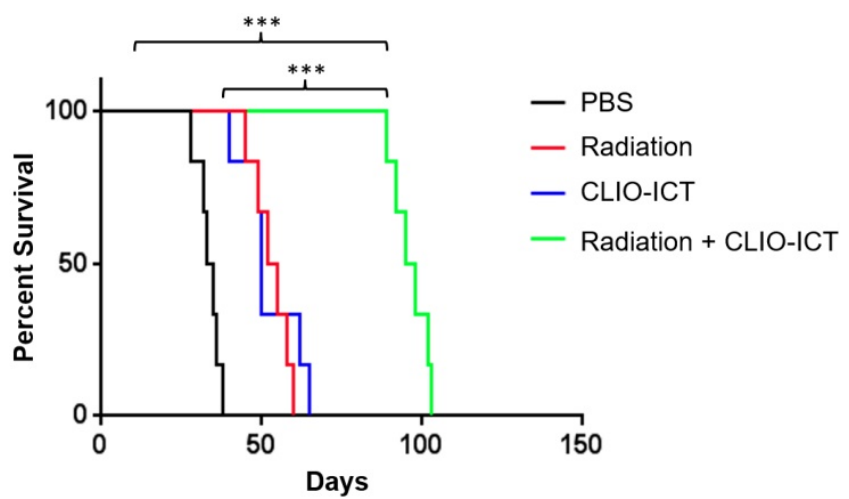

Figure 7: Radiation and CLIO-ICT combination therapy significantly improves survival of GBM39 tumor-bearing mice. Kaplan-Meier survival curves demonstrate a significant survival benefit in CLIO-ICT and radiotherapy co-treated group compared with monotherapy or vehicle control $(n=6)$, log-rank Mantel-Cox test, $* \mathrm{p}<0.05, * * \mathrm{p}<0.01,{ }^{*} * * \mathrm{p}<0.001, \mathrm{~ns}=$ not significant.

Vasculature staining with CD31 and desmin showed reduced vascular density for mice treated with combination therapy (Figure 6A \& 6B). The staining for CD15, a known marker for glioblastoma initiating cells [25], also dramatically decreased substantially upon the combination treatment compared to monotherapy and control groups (Figure $6 \mathrm{~A} \& 6 \mathrm{~B})$. Furthermore, the amount of active caspase-3 increased significantly in radiation and CLIO-ICT co-treated groups compared to the PBS treated control group ( $\mathrm{p}<0.001$ ), suggesting that apoptosis was induced by the former treatment (Figure 6A \& 6B). As expected, we also found that the combination therapy significantly prolonged the survival of GBM-bearing mice compared to control or monotherapy treated animals (Figure 7, p < 0.001). We used H\&E staining to confirm that the entire tissue section stained was tumor, which could be recognized by extensive hyperplasia (Supplementary Figure 4).

\section{Discussion}

Despite advances in therapy, the prognosis for GBM patients is still devastating. The recurrence rate of malignant gliomas is approximately 90\% [26]. Despite aggressive treatment with surgical resection and subsequent chemo/radiation therapy, resistance to adjuvant therapy enhances cancer cell survival, which is a critical cause for tumor relapse [3, 4, 27]. While effective for eradicating GBM cancer cells, radiotherapy is less effective for GICs [7, 28]. Radiation therapy kills cancer cells by inducing irreparable DNA double-strand breaks, thus highly 
proliferating cancer cells are more radiosensitive than quiescent GICs. Combining radiation therapy with other cancer therapies has shown benefits over monotherapies, including targeting multiple biochemical pathways, genes, or cell cycle checkpoints to overcome resistance in heterogeneous tumors [29, 30]. However, thus far, a curative combination therapy for GBM that can target GICs without added toxic effects has not been found.

Here we investigated a new combined therapeutic approach against GBMs using radiation and the MMP-14-activatable theranostic nanoparticle, CLIO-ICT. We found that the combination therapy demonstrated significant tumor response and prolonged survival time compared to monotherapy or no treatment. The data suggests that CLIO-ICT can improve the therapeutic efficacy of radiation therapy for GBM treatment. There are several examples in the literature where combination therapies involving nanoparticles have enhanced therapeutic outcomes without added toxicity, enhanced efficacy, and overcome drug resistance to elicit sustained treatment responses in cancer patients [30-32]. Given our promising results and the fact that CLIO-ICT is based on an FDA-approved iron oxide scaffold, this could potentially be a translatable treatment option for human GBMs.

The combination of CLIO-ICT with ionizing radiation is particularly potent because CLIO-ICT is activated through an MMP-14-cleavable linker and thereby, only activated in the tumor tissue. This limits off-target toxicities to the normal brain. MMP-14 is a membrane-bound protein that plays a role in the degradation of structural proteins in the extracellular matrix and has been identified as an important target for cancer diagnostics and therapy [13, 33]. In addition, MMP-14 is also regarded as a marker for GICs [13-15], which makes it a potential target for our drug. As expected, we found a significant reduction in CD15 staining (a marker for glioblastoma initiating cells) in CLIO-ICT and CLIO-ICT plus radiation treated mice, confirming that CLIO-ICT targets cells that have been previously reported as radio-resistant.

One concern with combination therapies is toxicity [34]. ICT is a derivative of colchicine, which is a VDA. VDAs are used currently in clinical practice, but they are highly toxic. For example, significant cardiotoxicity has been observed in patients treated with the VDAs DMXAA [35] and combretastatin A4 $[36,37]$. Colchicine is an effective VDA not only because it binds to tubulin and shuts down mitosis but also because it activates platelets which causes serotonin release. Serotonin is also a VDA, acting through G-protein-coupled receptors to generate actin stress fibers and decrease blood flow [38]. Our data shows that CLIO-ICT does not accumulate in the heart, because the ICT is covalently linked to nanoparticles which do not extravasate under normal physiological conditions. Our data are in accordance with our previous observations which showed no significant cardiotoxicity after CLIO-ICT treatment [11]. While we showed that our CLIO-ICT can potentiate the effect of radiation therapy, radiation therapy can also potentiate the efficacy of our drug. Previous investigators described that, radiation can cause an upregulation of MMPs [39, 40]. Greater expression of MMPs would result in enhanced CLIO-ICT activation in the tumor tissue.

VDAs have other advantages to overcome tumor resistance to radiotherapy. It is reported that larger tumors are less radiosensitive than small tumors, which might be due to increased hypoxic regions. VDAs cause a rapid shutdown of perfusion in the established tumor vasculature, leading to tumor cell ischemia and secondary tumor cell death [41-43]. These agents have the potential to destroy existing tumor masses and may, therefore, be particularly suitable for treating large tumors [44]. Complementarily, conventional cytotoxic radiotherapy has been shown to eradicate a viable rim of tumor cells at the tumor periphery after VDA treatment [45]. This observation might be explained by the fact that VDAs are able to kill cancer cells also in hypoxic regions which are less radiosensitive. The remaining rim of viable cells after mono-VDA treatment is believed to be well-oxygenated and thus present an excellent target for conventional cytotoxic radiotherapy. To achieve the strongest additive effect, we treated our animals first with radiotherapy and next with CLIO-ICT (at 3 hours post-radiotherapy), since it has been suggested that blood flow needs to be reestablished to obtain maximum radiosensitization of the tumor [46].

\section{Conclusion}

In summary, intravenous treatment with theranostic CLIO-ICT nanoparticles enhanced the efficacy of ionizing radiation for the treatment of GBM in mouse models in an additive manner, with limited toxicity to the normal brain or visceral organs. In addition, the use of superparamagnetic iron oxide nanoparticles as the backbone of our theranostic drug enabled in vivo drug tracking with MRI. Given the urgent need for new therapies and the shortcomings of current clinical approaches, this new combination therapy provides a new option for GBM treatment. Further studies to enable clinical translation of CLIO-ICT are under way. 


\section{Abbreviations}

Glioblastoma multiforme (GBM), glioblastoma initiating cell (GIC), cross-linked iron oxide nanoparticle (CLIO), matrix-metalloproteinase (MMP), vasculature disrupting agent (VDA), microvessel density (MVD), enhanced permeability and retention (EPR), magnetic resonance imaging (MRI), bioluminescence imaging (BLI), computed tomography (CT), azademethylcolchicine-peptide conjugate (ICT2588), modified analogue of ICT2588 (ICT), 5,6-dimethylxanthenone-4-acetic acid (DMXAA).

\section{Supplementary Material}

Supplementary figures.

http://www.ntno.org/v03p0299s1.pdf

\section{Acknowledgements}

We thank the Small Animal Imaging Facility at Stanford for providing the equipment and infrastructure for this project. We thank members of the Daldrup-Link, Gambhir, Graves, and ICT Bradford laboratories for valuable discussions. This work was in part supported by a SPARK Translational Research Program Grant to HEDL, an $\mathrm{NIH} / \mathrm{NCI}$ training grant (T32 CA118681) to JLK and a CCNE-TD U54 grant from the National Cancer Institute (1U54CA199075) to Dr. Sanjiv Sam Gambhir. A University of Bradford Commercial Development Fund award (to RAF) provided support towards the costs associated with chemical synthesis of the ICT drug.

\section{Author contributions}

WW, JLK, SuM, and HEDL designed the experiments, with input from StM, JS, EEG, ZC, JR, PML and RAF. SuM, WW, JLK, KSK, and NZ performed the in vitro and in vivo experiments. SuM, KL, ZC, GRM, and RAF synthesized ICT and CLIO-ICT. SuM, WW, JLK, and HEDL analyzed the data. SuM and HEDL wrote the manuscript. WW, JLK, JS, EEG, RAF, PML, FTC, and HEDL edited the manuscript and approved the final version.

\section{Competing Interests}

HEDL, RAF, and PML hold a joint patent on the described theranostic nanoparticles (WO 2015014756).

\section{References}

1. Prados MD, Byron SA, Tran NL, Phillips JJ, Molinaro AM, Ligon KL, et al. Toward precision medicine in glioblastoma: the promise and the challenges. Neuro Oncol. 2015; 17: 1051-63.

2. Jue TR, McDonald KL. The challenges associated with molecular targeted therapies for glioblastoma. J Neurooncol. 2016; 127: 427-34.

3. Beauchesne P, Quillien V, Faure G, Bernier V, Noel G, Quetin P, et al. A concurrent ultra-fractionated radiation therapy and temozolomide treatment:
A promising therapy for newly diagnosed, inoperable glioblastoma. Int J Cancer. 2016; 138: 1538-44

4. Cohen KJ, Pollack IF, Zhou T, Buxton A, Holmes EJ, Burger PC, et al. Temozolomide in the treatment of high-grade gliomas in children: a report from the Children's Oncology Group. Neuro Oncol. 2011; 13: 317-23.

5. Cohen MH, Johnson JR, Pazdur R. Food and Drug Administration Drug approval summary: temozolomide plus radiation therapy for the treatment of newly diagnosed glioblastoma multiforme. Clin Cancer Res. 2005; 11: 6767-71.

6. Frosina G. DNA repair and resistance of gliomas to chemotherapy and radiotherapy. Mol Cancer Res. 2009; 7: 989-99.

7. Bao $S, W u$ Q, McLendon RE, Hao $Y$, Shi $\mathrm{O}$, Hjelmeland $\mathrm{AB}$, et al. Glioma stem cells promote radioresistance by preferential activation of the DNA damage response. Nature. 2006; 444: 756-60.

8. Krause M, Dubrovska A, Linge A, Baumann M. Cancer stem cells: Radioresistance, prediction of radiotherapy outcome and specific targets for combined treatments. Adv Drug Deliv Rev. 2017; 109: 63-73.

9. Rieger KE, Hong WJ, Tusher VG, Tang J, Tibshirani R, Chu G. Toxicity from radiation therapy associated with abnormal transcriptional responses to DNA damage. Proc Natl Acad Sci U S A. 2004; 101: 6635-40.

10. Parhi P, Mohanty C, Sahoo SK. Nanotechnology-based combinational drug delivery: an emerging approach for cancer therapy. Drug Discov Today. 2012; 17: 1044-52.

11. Mohanty S, Chen Z, Li K, Morais GR, Klockow J, Yerneni K, et al. A Novel Theranostic Strategy for MMP-14-Expressing Glioblastomas Impacts Survival. Mol Cancer Ther. 2017; 16: 1909-21.

12. Ansari C, Tikhomirov GA, Hong SH, Falconer RA, Loadman PM, Gill JH, et al. Development of novel tumor-targeted theranostic nanoparticles activated by membrane-type matrix metalloproteinases for combined cancer magnetic resonance imaging and therapy. Small. 2014; 10: 566-75, 417

13. Ulasov I, Yi R, Guo D, Sarvaiya P, Cobbs C. The emerging role of MMP14 in brain tumorigenesis and future therapeutics. Biochim Biophys Acta. 2014; 1846: 113-20.

14. Knapinska AM, Fields GB. The Expanding Role of MT1-MMP in Cancer Progression. Pharmaceuticals (Basel). 2019; 12

15. Hillebrand LE, Wickberg SM, Gomez-Auli A, Follo M, Maurer J, Busch H, et al. MMP14 empowers tumor-initiating breast cancer cells under hypoxic nutrient-depleted conditions. FASEB J. 2019; 33: 4124-40.

16. Nakada $\mathrm{M}$, Nakamura $\mathrm{H}$, Ikeda $\mathrm{E}$, Fujimoto $\mathrm{N}$, Yamashita $\mathrm{J}$ Sato $\mathrm{H}$, et al. Expression and tissue localization of membrane-type 1, 2, and 3 matrix metalloproteinases in human astrocytic tumors. Am J Pathol. 1999; 154: 417-28.

17. Nakada M, Kita D, Futami K, Yamashita J, Fujimoto N, Sato H, et al. Roles of membrane type 1 matrix metalloproteinase and tissue inhibitor of metalloproteinases 2 in invasion and dissemination of human malignant glioma. J Neurosurg. 2001; 94: 464-73.

18. Munaut $\mathrm{C}$, Noel A, Hougrand O, Foidart JM, Boniver J, Deprez M. Vascular endothelial growth factor expression correlates with matrix metalloproteinases MT1-MMP, MMP-2 and MMP-9 in human glioblastomas. Int J Cancer. 2003; 106: 848-55.

19. Hagemann C, Anacker J, Ernestus RI, Vince GH. A complete compilation of matrix metalloproteinase expression in human malignant gliomas. World $\mathrm{J}$ Clin Oncol. 2012; 3: 67-79.

20. Chang E, Pohling C, Natarajan A, Witney TH, Kaur I, Xu L, et al. AshwaMAX and Withaferin A inhibits gliomas in cellular and murine orthotopic models. J Neurooncol. 2016; 126: 253-64.

21. Graves EE, Quon A, Loo BW, Jr. RT Image: an open-source tool for investigating PET in radiation oncology. Technol Cancer Res Treat. 2007; 6: $111-21$

22. Lemasson B, Wang H, Galban S, Li Y, Zhu Y, Heist KA, et al. Evaluation of Concurrent Radiation, Temozolomide and ABT-888 Treatment Followed by Maintenance Therapy with Temozolomide and ABT-888 in a Genetically Engineered Glioblastoma Mouse Model. Neoplasia. 2016; 18: 82-9.

23. Atkinson JM, Falconer RA, Edwards DR, Pennington CJ, Siller CS, Shnyder $\mathrm{SD}$, et al. Development of a novel tumor-targeted vascular disrupting agent activated by membrane-type matrix metalloproteinases. Cancer Res. 2010; 70: 6902-12.

24. Bronte V, Pittet MJ. The spleen in local and systemic regulation of immunity. Immunity. 2013; 39: 806-18

25. Mao XG, Zhang X, Guo G, Wang P, Zhang W, Fei Z, Zhen HN, You SW, Yang H. Brain tumor stem-like cells identified by neural stem cell marker CD15. Transl Oncol. 2009; 2: 247-57.

26. Ringel F, Pape H, Sabel M, Krex D, Bock HC, Misch M, et al. Clinical benefit from resection of recurrent glioblastomas: results of a multicenter study including 503 patients with recurrent glioblastomas undergoing surgical resection. Neuro Oncol. 2016; 18: 96-104.

27. Osuka S, Van Meir EG. Overcoming therapeutic resistance in glioblastoma: the way forward. J Clin Invest. 2017; 127: 415-26.

28. Caragher SP, Sachdev S, Ahmed A. Radiotherapy and Glioma Stem Cells: Searching for Chinks in Cellular Armor. Curr Stem Cell Rep. 2017; 3: 348-57.

29. Baskar R, Lee KA, Yeo R, Yeoh KW. Cancer and radiation therapy: current advances and future directions. Int J Med Sci. 2012; 9: 193-9.

30. Gurunathan S, Kang MH, Qasim M, Kim JH. Nanoparticle-Mediated Combination Therapy: Two-in-One Approach for Cancer. Int J Mol Sci. 2018;

31. Jadia R, Scandore C, Rai P. Nanoparticles for Effective Combination Therapy of Cancer. Int J Nanotechnol Nanomed. 2016; 1 . 
32. He C, Chan C, Weichselbaum RR, Fleming GF, Yamada SD, Lin W. Nanomedicine for Combination Therapy of Cancer. EBioMedicine. 2015; 2: 366-7.

33. Malemud CJ. Matrix metalloproteinases (MMPs) in health and disease: an overview. Front Biosci. 2006; 11: 1696-701.

34. Belgioia L, Desideri I, Errico A, Franzese C, Daidone A, Marino L, et al. Safety and efficacy of combined radiotherapy, immunotherapy and targeted agents in elderly patients: A literature review. Crit Rev Oncol Hematol. 2019; 133: 163-70.

35. McKeage MJ, Fong P, Jeffery M, Baguley BC, Kestell P, Ravic M, et al. 5,6-Dimethylxanthenone-4-acetic acid in the treatment of refractory tumors: a phase I safety study of a vascular disrupting agent. Clin Cancer Res. 2006; 12: 1776-84.

36. Cooney MM, Radivoyevitch T, Dowlati A, Overmoyer B, Levitan N, Robertson $\mathrm{K}$, et al. Cardiovascular safety profile of combretastatin a4 phosphate in a single-dose phase I study in patients with advanced cancer. Clin Cancer Res. 2004; 10: 96-100.

37. Rustin GJ, Galbraith SM, Anderson H, Stratford M, Folkes LK, Sena L, et al. Phase I clinical trial of weekly combretastatin A4 phosphate: clinical and pharmacokinetic results. J Clin Oncol. 2003; 21: 2815-22.

38. Tozer GM, Kanthou C, Baguley BC. Disrupting tumour blood vessels. Nat Rev Cancer. 2005; 5: 423-35.

39. Heo W, Lee YS, Son CH, Yang K, Park YS, Bae J. Radiation-induced matrix metalloproteinases limit natural killer cell-mediated anticancer immunity in NCI-H23 lung cancer cells. Mol Med Rep. 2015; 11: 1800-6.

40. Ulasov I, Thaci B, Sarvaiya P, Yi R, Guo D, Auffinger B, et al. Inhibition of MMP14 potentiates the therapeutic effect of temozolomide and radiation in gliomas. Cancer Med. 2013; 2: 457-67.

41. Huang X, Molema G, King S, Watkins L, Edgington TS, Thorpe PE. Tumor infarction in mice by antibody-directed targeting of tissue factor to tumor vasculature. Science. 1997; 275: 547-50.

42. Dark GG, Hill SA, Prise VE, Tozer GM, Pettit GR, Chaplin DJ. Combretastatin A-4, an agent that displays potent and selective toxicity toward tumor vasculature. Cancer Res. 1997; 57: 1829-34.

43. Hollebecque A, Massard C, Soria JC. Vascular disrupting agents: a delicate balance between efficacy and side effects. Curr Opin Oncol. 2012; 24: 305-15.

44. Siemann DW, Bibby MC, Dark GG, Dicker AP, Eskens FA, Horsman MR, et al. Differentiation and definition of vascular-targeted therapies. Clin Cancer Res. 2005; 11: 416-20.

45. Nishiguchi I, Willingham V, Milas L. Tumor necrosis factor as an adjunct to fractionated radiotherapy in the treatment of murine tumors. Int J Radiat Oncol Biol Phys. 1990; 18: 555-8.

46. Sersa G, Willingham V, and Milas L., Anti-tumor effects of tumor necrosis factor alone or combined with radiotherapy. International Journal of Cancer, 1988, 42(1):129-134. 